\section{Diseño y validación del instrumento SALUFAM: un instrumento de valoración de la salud familiar con alto valor predictivo clínico para la atención primaria chilena}

\author{
KLAUS PUSCHEL ${ }^{1, \mathrm{a}}$, PAULA REPETTO ${ }^{2, \mathrm{~b}, \mathrm{c}}$, MARÍA OLGA SOLAR ${ }^{3, \mathrm{~d}}$, \\ GABRIELA SOTO ${ }^{1, c}$, KARLA GONZÁLEZ $^{1, c}$
}

\section{Design and validation of an instrument to assess families at risk for health problems}

Background: There is a paucity of screening instruments with a high clinical predictive value to identify families at risk and therefore, develop focused interventions in primary care. Aim: To develop an easy to apply screening instrument with a high clinical predictive value to identify families with a higher health vulnerability. Material and Methods: In the first stage of the study an instrument with a high content validity was designed through a review of existent instruments, qualitative interviews with families and expert opinions following a Delphi approach of three rounds. In the second stage, concurrent validity was tested through a comparative analysis between the pilot instrument and a family clinical interview conducted to 300 families randomly selected from a population registered at a primary care clinic in Santiago. The sampling was blocked based on the presence of diabetes, depression, child asthma, behavioral disorders, presence of an older person or the lack of previous conditions among family members. The third stage, was directed to test the clinical predictive validity of the instrument by comparing the baseline vulnerability obtained by the instrument and the change in clinical status and health related quality of life perceptions of the family members after nine months of follow-up. Results: The final SALUFAM instrument included 13 items and had a high internal consistency (Cronbach's alpha: 0.821), high test re-test reproducibility (Pearson correlation: 0.84) and a high clinical predictive value for clinical deterioration (Odds ratio: 1.826; 95\% confidence intervals: 1.101-3.029). Conclusions: SALUFAM instrument is applicable, replicable, has a high content validity, concurrent validity and clinical predictive value.

(Rev Med Chile 2012; 140: 417-425).

Key words: Family characteristics; Healthcare surveys; Predictive value of tests.

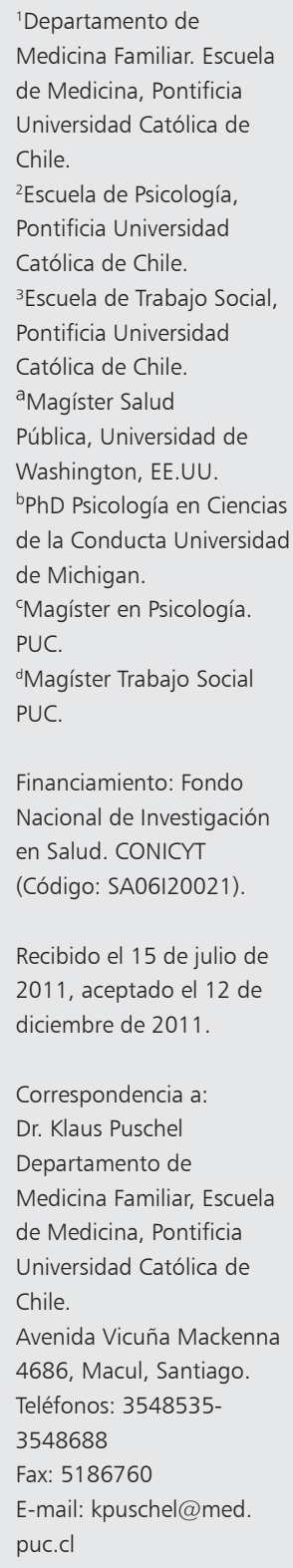

L a evidencia existente demuestra que países con una atención primaria "más fuerte" alcanzan mejores indicadores de salud a un menor gasto per cápita ${ }^{1}$. Uno de los elementos esenciales de una atención primaria "fuerte" es la existencia del enfoque familiar en su modelo de atención ${ }^{2}$. Diversos estudios muestran que la familia tiene una importante influencia en el de- sarrollo y control de problemas de salud frecuentes en la atención primaria de salud (APS) tales como trastornos conductuales en niños ${ }^{3,4}$, depresión en adolescentes $^{5}$, asma infantil ${ }^{6}$, problemas cardiovasculares ${ }^{7}$ y control de diabetes mellitus tipo II $^{8}$.

La aplicación eficiente y efectiva del modelo de salud familiar requiere contar con instrumentos que permitan identificar en forma simple y precisa 
aquellos grupos familiares con mayor y menor riesgo de desarrollar complicaciones en la salud de sus miembros?.

En Chile, se han utilizado diversos instrumentos para determinar las interrelaciones y el funcionamiento de una familia. Ellos se han utilizado mayoritariamente como apoyo diagnóstico y no como método de tamizaje para focalizar intervenciones

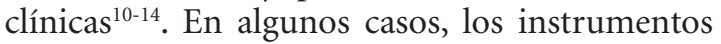
disponibles presentan una alta inestabilidad en el tiempo, carecen de validez clínica predictiva o han sido desarrollados hace varias décadas y pueden no representar apropiadamente la situación de salud familiar y de la atención primaria actual ${ }^{12-14,15}$.

Este estudio tiene como objetivo central el diseñar y validar un instrumento de tamizaje de vulnerabilidad familiar en salud primaria en Chile. La investigación buscó obtener un instrumento aplicable en los centros de atención primaria que permitiera identificar en forma precisa dimensiones de funcionamiento familiar que se asociaran con una mejor o peor evolución de problemas clínicos frecuentes y que fueran susceptibles de intervenir por los equipos de salud.

\section{Material y Métodos}

La investigación contempló tres etapas que son presentadas en la Figura 1. La primera etapa de diseño estuvo dirigida a obtener un instrumento con alta validez de contenido y se desarrolló bajo tres perspectivas. En la "perspectiva científica" se realizó una revisión de instrumentos de valoración familiar publicados en la literatura nacional e internacional a partir del año 1970 en bases de datos primarias (PubMed/Embase/psychoinfo/LILACS), textos clásicos relacionados con el tema ${ }^{16-18}$ y documentos aportados por expertos del Ministerio de Salud ${ }^{19,20}$. Se seleccionaron cuatro instrumentos de valoración familiar en base al cumplimiento de cinco criterios esenciales: contenido (instrumento que evaluara vulnerabilidad familiar), validación de criterio concurrente (evaluado como tamizaje frente a gold standard), validación en Chile, predictibildad clínica y aplicabilidad (Tabla 1). En la "perspectiva de los usuarios" se desarrolló una metodología cualitativa que utilizó un muestreo a propósito no probabilístico que permitiera la selección de familias de diferente estructura y condiciones de salud en sus miembros. Se seleccionaron quince familias que incluían familias nucleares, extendidas, monoparentales, ensambladas, y unipersonales. Dentro de ellas, se seleccionaron familias en los que al menos uno de sus miembros tuviera una enfermedad crónica (diabetes mellitus o hipertensión arterial), trastorno depresivo, asma infantil, trastorno por déficit atencional o que contara entre sus integrantes con un adulto mayor. La metodología de diseño cualitativo utilizada fue la de modelo de marco analítico ${ }^{21}$. Los grupos

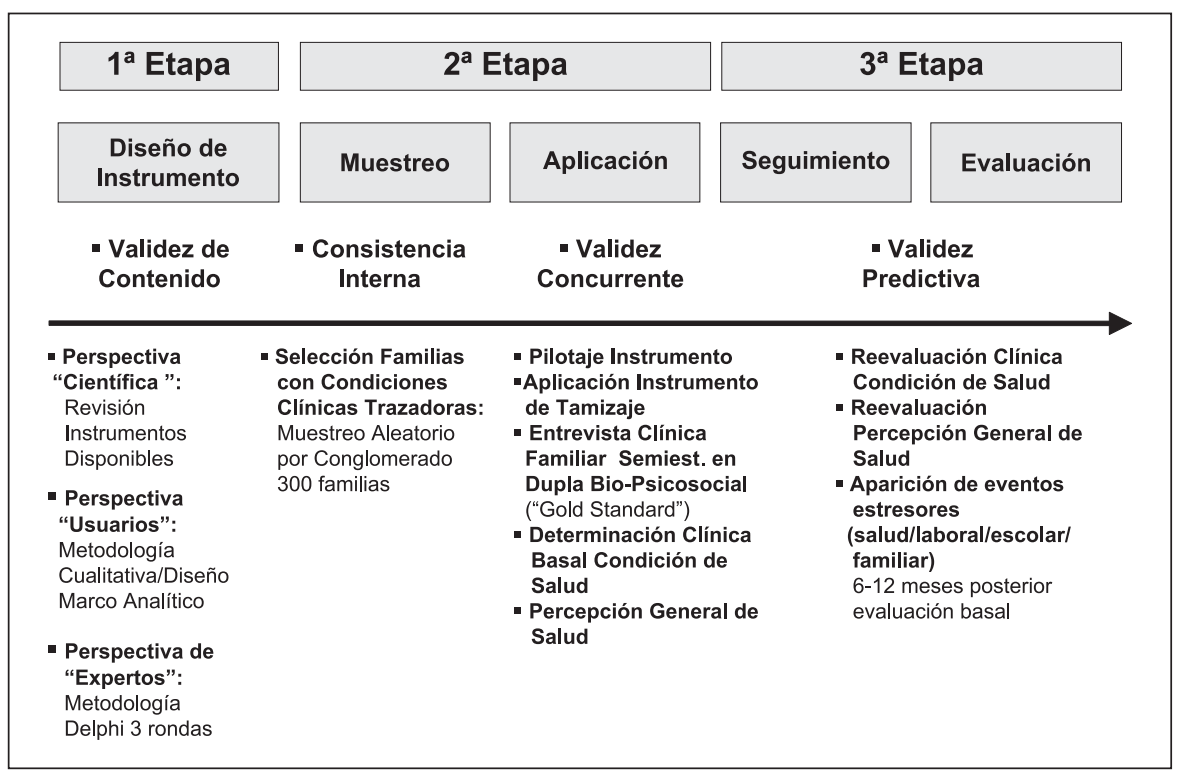

Figura 1. Etapas del estudio de diseño y validación del instrumento SALUFAM. 
focales fueron realizados por dos profesionales con experiencia en metodología cualitativa. Todas las entrevistas fueron grabadas y luego transcritas completamente para su análisis. El análisis de la información se realizó mediante codificación abierta, axial y selectiva.

Finalmente, la "perspectiva de expertos" contempló el uso de una metodología Delphi de tres rondas en la que un panel de 10 expertos en familia y salud evaluó y jerarquizó las dimensiones e ítems centrales que debiera contener un instrumento de valoración familiar. La selección del panel de expertos fue por conveniencia y se aplicó la técnica de la "bola de nieve". Esta técnica utiliza un muestreo dinámico basado en la selección de actores claves que surgen de la información aportada en forma progresiva por cada participante. La técnica fue de especial utilidad para obtener participantes de diversas disciplinas y reconocida trayectoria capaces de entregar validez de contenido al instrumento

Tabla 1. Instrumentos de valoración familiar utilizados en atención primaria en Chile

\begin{tabular}{|c|c|c|c|c|c|}
\hline Instrumento (Ref.) & Apgar (13) & EFF (14) & CAF (11) & TU/SU (12) & SALUFAM* \\
\hline Validación en Chile & Sí & Sí & Sí & Sí & \\
\hline Tamizaje en APS (validez de criterio concurrente) & Sí & No & No & Sí & \\
\hline Validez Predictiva APS & No & No & No & No & \\
\hline N de Itemes (Aplicabilidad) & 5 & 21 & 19 & 135 & $65^{*}$ \\
\hline \multicolumn{6}{|l|}{ Factores familiares evaluados } \\
\hline Acuerdo & & + & & & + \\
\hline Adaptabilidad & + & & + & & \\
\hline Afecto & + & & & & + \\
\hline Afrontamiento & & + & & + & + \\
\hline Ciclo Vital & & & & + & + \\
\hline Cohesión & & + & + & + & + \\
\hline Colaboración & + & & & & \\
\hline Comunicación & & & & + & \\
\hline Compromiso & & + & & & + \\
\hline Crecimiento & + & & & & \\
\hline Estructura Familiar & & & & + & + \\
\hline Fortaleza Familiar & & + & & & \\
\hline Jerarquías & & & & + & \\
\hline Participación & & & & + & \\
\hline Recursos Internos & & + & & + & \\
\hline Red de Apoyo & & + & & + & + \\
\hline Resolución & + & & & & \\
\hline Rutinas & & & & + & \\
\hline Satisfacción & & & & + & \\
\hline Tensiones & & & & + & \\
\hline Salud & & & & + & + \\
\hline Valores & & & & + & \\
\hline
\end{tabular}

Apgar: "Adaptability" (adaptabilidad), "partnership" (colaboración), "growth" (crecimiento), "affection" (afectos), "resolve" (resolución). EFF: Escala de Funcionamiento Familiar. CAF: Cohesión y Adaptabilidad Familiar. SALUFAM* Versión piloto inicial. 
desarrollado. Este conjunto de expertos pertenecía a diversas instituciones tales como Universidad de Chile, Universidad de Concepción, Pontificia Universidad Católica de Chile, Centro Nacional de la Familia, Unicef, Fundación de Salud el Teniente, Municipalidad de La Pintana. El cuestionario inicial se elaboró en base a la información recogida de las dos perspectivas previas.

La segunda etapa del estudio estuvo dirigida a obtener un instrumento de alta consistencia interna y validez concurrente. En esta fase se aplicó el instrumento inicial a un conjunto de familias a las que además, se realizó una entrevista clínicafamiliar en profundidad. La selección de familias se realizó mediante un muestreo aleatorio por conglomerado de 300 familias residentes en el sector de El Castillo, La Pintana, que hubieran tenido al menos una consulta en atención primaria y por tanto, que estuvieran registradas en la base de datos electrónica del Centro de Salud Familiar Juan Pablo II en La Pintana. Cada conglomerado de familias fue definido utilizando la metodología de condiciones trazadoras. Se buscaron condiciones que fueran relevantes (alta prevalencia en atención primaria y alto impacto en salud), identificables, intervenibles, asociadas a variables familiares y representativas, en su conjunto, del ciclo vital. La definición de condición trazadora se realizó en base a la Clasificación Internacional de Enfermedades para Atención Primaria (CIAP). Las condiciones seleccionadas fueron "Diabetes Mellitus" (CIAP: T90); “Trastorno Depresivo Mayor" (CIAP P73P76); “Asma Infantil” (R 91-R96) y "Trastorno por Déficit Atencional” (P21-P22-P24). Además se incorporó la condición "Funcionalidad de Adulto Mayor" y "Sin Condición". En este último caso se seleccionaron familias en donde no existiera ningún integrante con alguna de las condiciones previamente descritas. Cada conglomerado estuvo conformado por 50 familias que tenían a uno de sus miembros con la condición descrita ("caso índice").

El tamaño muestral para desarrollar el proceso de validación concurrente del instrumento consideró un nivel de confianza de $95 \%$ para obtener una muestra representativa del total de familias de El Castillo. Además, se estimó el tamaño muestral requerido para el proceso de validación predictiva del instrumento que basado en el seguimiento de familias de diverso grado de vulnerabilidad en salud. Se estimó una diferencia de vulnerabilidad en salud (alta vs baja) de $40 \%$, poder de $80 \%$ (beta $20 \%$ ) y un valor alfa de dos colas de $5 \%$. Con estos parámetros el número requerido de familias a evaluar y seguir fue de 253. Se estimó, además, un nivel de atrición de la muestra (en la fase de seguimiento) de $10 \%$ con lo cual el número requerido fue de 269 familias. Para ampliar el poder del estudio y dado las dificultades de seguimiento potenciales en familias de alta vulnerabilidad social se decidió incorporar a 300 familias.

Un representante de las familias seleccionadas respondió el instrumento en forma autoaplicada y luego participó junto a un acompañante en una entrevista clínica familiar. Esta fue realizada por un dupla de profesionales bio-psicosocial (médico-enfermera/psicóloga-trabajadora social) previamente capacitados y siguiendo una pauta semiestructurada.

La evaluación inicial de los participantes incluyó la aplicación de una serie de mediciones para determinar la condición clínica del "caso índice", la existencia de eventos vitales de estrés familiar ${ }^{22}$, y la percepción general de salud ${ }^{23}$. Los parámetros de valoración clínica utilizados fueron los siguientes: nivel de $\mathrm{Hb}$ Glicosilada Ac 1, Escala de Depresión de $\mathrm{Beck}^{24}$, Cuestionario de Síntomas de Asma ${ }^{25}$, Escala de Valoración de Ackenbach ${ }^{26}$ y Escala de Evaluación Funcional del Adulto Mayor ${ }^{27}$. Las evaluaciones clínicas se realizaron al inicio y al final del período de seguimiento.

La tercera etapa consistió en el seguimiento de las familias y su reevaluación clínica. Este proceso permitió determinar la validez de criterio predictiva del instrumento. El análisis estadístico final se basó en la aplicación de un modelo predictivo multivariado. El instrumento obtenido se volvió a aplicar a una muestra de $10 \%$ de familias participantes para determinar su replicabilidad test re-test. Además se evaluó la aplicabilidad del instrumento en base a tiempo de aplicación y comprensión de los ítems incluidos.

Este estudio fue revisado y aprobado por el Comité de Ética del Centro de Investigaciones Médicas de la Pontificia Universidad Católica de Chile.

\section{Resultados}

La Tabla 1 presenta una comparación de los factores incluidos en las diferentes escalas de valoración existentes y el instrumento SALUFAM 
inicial. Destaca la variabilidad en la extensión de las escalas existentes y la falta de validez predictiva en APS.

La Tabla 2 presenta las características generales y por condición de salud de las 300 familias participantes en el estudio. Puede observarse que predominaban familias nucleares y monoparentales de bajo nivel socioeconómico.

En la evaluación cualitativa participaron 30 personas y se registró un total de 291 minutos de grabación. En el análisis de la información surgieron tres categorías principales. La primera siguió el eje de la relación-barrio-familia y salud. La segunda categoría fue la familia como sistema cohesionado y protectora de la salud en contraste con la familia disgregada y las personas aisladas como factor de riesgo para la salud. Uno de los principales tópicos en esta categoría fue el "conta- gio" en las conductas y estados de ánimo entre los miembros de la familia y la capacidad de la familia para generar acuerdos frente a situaciones difíciles o estresantes. La tercera categoría se centró en la relación red de apoyo-aislamiento-salud-enfermedad. Aquí se resaltó la capacidad de la familia para generar redes de apoyo con familiares, vecinos o instituciones y su efecto en facilitar el cuidado en salud de los miembros.

El análisis factorial de consistencia interna del instrumento piloto incluyó 12 subescalas que incluían las dimensiones de acuerdo, cohesión, expresión emocional, conflicto, compromiso, confianza, apoyo social, protección de salud, barrio, estresores laborales, estresores familiares y estresores de salud. Estas dimensiones contenían un total de 65 ítems (Tabla 1). Para la selección final de los ítems, se consideraron aquellos contenidos

Tabla 2. Características de los participantes en el estudio

\begin{tabular}{|c|c|c|c|c|c|c|}
\hline \multirow{2}{*}{\multicolumn{2}{|c|}{$\begin{array}{l}\text { Características generales } \\
\text { de las familias } \\
\text { Variable }\end{array}$}} & \multicolumn{5}{|c|}{ Características clínicas de los participantes por condición } \\
\hline & & Condición & $\begin{array}{l}\text { n partici- } \\
\text { pantes }\end{array}$ & $\begin{array}{l}\text { Edad } \\
\text { (DE) }\end{array}$ & $\begin{array}{c}\% \\
\text { mujeres }\end{array}$ & $\begin{array}{l}\text { Perfil Clínico Basal } \\
\text { Promedio (X) y (DE) }\end{array}$ \\
\hline n total de familias & 300 & $\begin{array}{l}\text { Diabetes } \\
\text { Mellitus II }\end{array}$ & 50 & $53,7(9,5)$ & 85 & $\begin{array}{l}\text { - Nivel de } \mathrm{HbA} 1 \mathrm{c} \\
\mathrm{X}=8,29 \mathrm{mg} / \mathrm{dl}(2,24) \\
<8,0 \mathrm{mg} / \mathrm{dl}: 50 \% \\
>/=8,0 \mathrm{mg} / \mathrm{dl}: 50 \%\end{array}$ \\
\hline $\begin{array}{lr}\text { Edad promedio (años } \\
\text { Caso índice (DE) } & 37,6 \\
\text { Acompañante (DE) } & 40,9\end{array}$ & $\begin{array}{l}\text { ) } \\
(25,1) \\
(27,2)\end{array}$ & T. depresivo & 50 & $42.7(14.6)$ & 92 & $\begin{array}{l}\text { - Puntaje escala depresión } \\
\text { X=30,7 (8.97) } \\
\text { Leve: } 10 \% \\
\text { Moderada: } 60 \% \\
\text { Severo: } 30 \%\end{array}$ \\
\hline $\begin{array}{l}\text { Sexo femenino } \\
\text { Caso índice }(\%) \\
\text { Acompañante }\end{array}$ & $\begin{array}{l}72 \\
66\end{array}$ & $\begin{array}{l}\text { Funcionalidad } \\
\text { adulto mayor }\end{array}$ & 50 & $71.8(8.1)$ & 76 & $\begin{array}{l}\text { - Puntaje EFAM } \\
X=42,2(2,98) \\
</=42: 50 \% \\
>42: 50 \%\end{array}$ \\
\hline $\begin{array}{lr}\text { Ingreso promedio } \\
\text { \$Chilenos } \\
\text { Familiar (DE) }\end{array}$ & $\begin{array}{l}4.233 \\
2.466)\end{array}$ & Asma & 50 & $7.04(4.2)$ & 50 & $\begin{array}{l}\text { Puntaje escala síntomas } \\
\text { X=53,2 (43.26) } \\
\text { Leve: } 20 \% \\
\text { Moderada: } 60 \% \\
\text { Severa: } 20 \%\end{array}$ \\
\hline $\begin{array}{l}\text { Escolaridad } \\
\text { Promedio adultos } \\
\mathrm{N} \text { de años de estudio (DE) }\end{array}$ & $\begin{array}{l}7.44 \\
(3.5)\end{array}$ & $\begin{array}{l}\text { Sd. déficit } \\
\text { atencional }\end{array}$ & 50 & $10.5(2.7)$ & 40 & $\begin{array}{l}\text { - Puntaje escala Achenbach } \\
\text { CBCL } \\
X=27,4(11,2)\end{array}$ \\
\hline $\begin{array}{l}\text { Tipo de Familia (\%)* } \\
\text { Nuclear } \\
\text { Extendida } \\
\text { Monoparental } \\
\text { Unipersonal }\end{array}$ & $\begin{array}{r}58 \\
42 \\
54 \\
3\end{array}$ & $\begin{array}{l}\text { Sin condi- } \\
\text { ción }\end{array}$ & 50 & $39.1(12.6)$ & 90 & $\begin{array}{l}\text { - Puntaje (\%) calidad de } \\
\text { vida salud escala Euroqol } \\
5 D \\
X=60,7(25,02)\end{array}$ \\
\hline
\end{tabular}

*Categorías no excluyentes. 
en las dimensiones con un alfa de Cronbach igual o superior a 0,8, eliminándose aquellos que en el re-análisis factorial no alcanzaran este nivel. Finalmente, las dimensiones de acuerdo $y$ apoyo social fueron las que mostraron mayor consistencia interna.

La Figura 2 presenta la validez de criterio concurrente estimada por la sensibilidad y especificidad de la escala SALUFAM final de 13 ítems, conteniendo las dimensiones de acuerdo y apoyo, a diferentes puntos de corte. Al compararla con la entrevista familiar, la escala SALUFAM obtiene $83 \%$ de sensibilidad

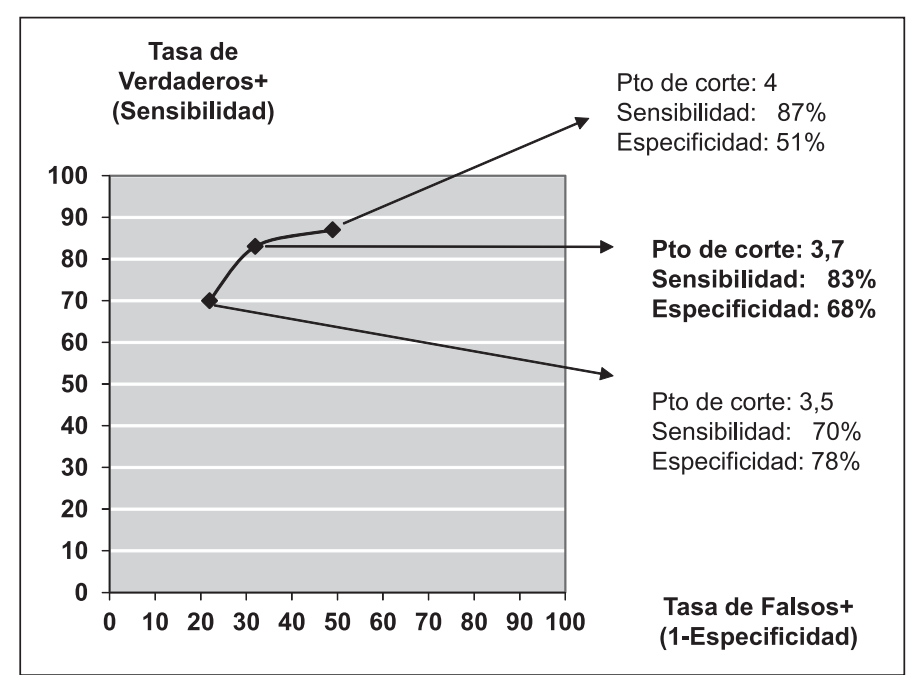

Figura 2. Estimación de la sensibilidad y especificidad de la escala SALUFAM final a diferentes puntos de corte. y $68 \%$ de especificidad a un punto de corte igual o menor a 3,7 .

El 93\% (280/300) de las familias completaron el seguimiento clínico a los 9 meses de la evaluación basal. En el modelo final de análisis se ajustaron las variables dependientes a un puntaje " $z$ " representando así a las condiciones basales ( $\mathrm{z} 1)$ y finales (z2) y su variación (z1 vs z2). El puntaje " $z$ " ajusta los parámetros clínicos asignando un valor estandarizado a cada uno de ellos dependiendo de si se observa o no un cambio y en qué dirección en la variable dependiente. Las funciones (F) de cada subescala y los errores E de cada ítem de las subescalas fueron incorporados al modelo estadístico final que permitió obtener un índice comparativo de ajuste (Comparative Fit Index [CFI]) que indica la proporción de la varianza explicada por el modelo y el grado de aproximación a un modelo de "calce" perfecto. En el modelo estudiado la escala SALUFAM obtuvo un CFI de 0,91 considerado como muy bueno. La Figura 3 presenta el resultado del modelo final del análisis basado en la

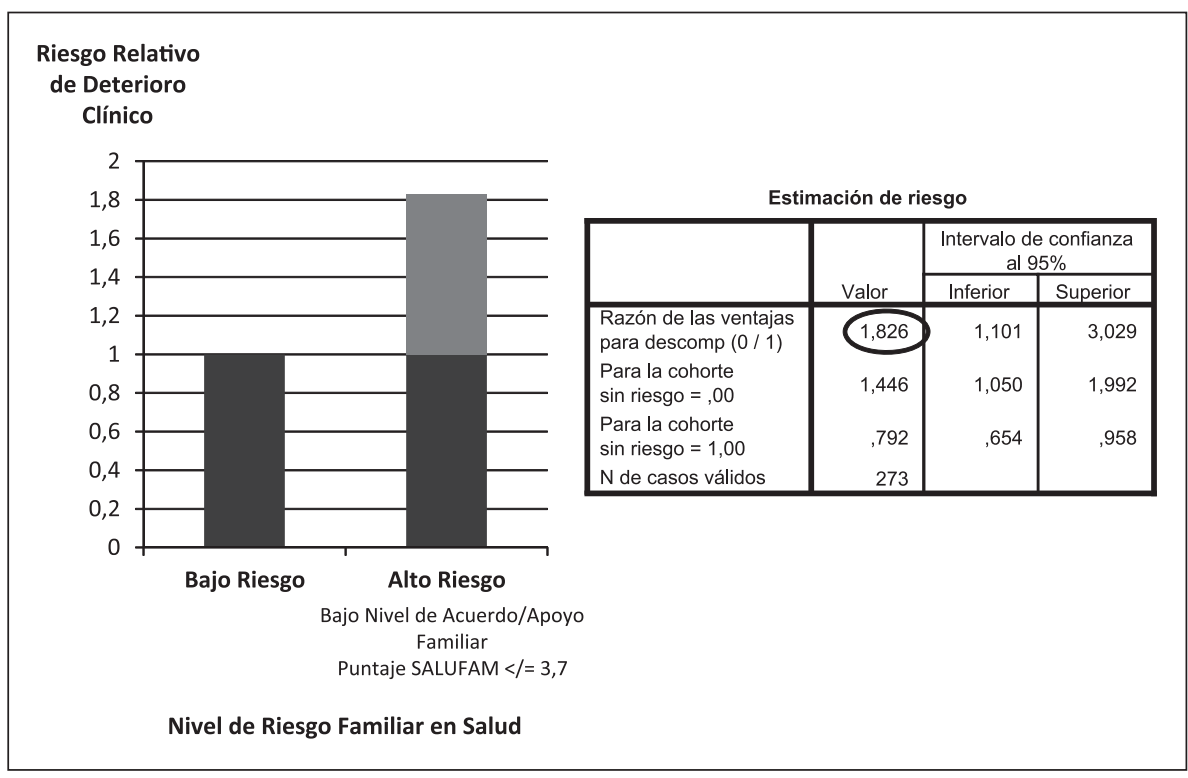

Figura 3. Riesgo relativo de deterioro clínico en relación a nivel de riesgo familiar. 
Tabla 3. Escala SALUFAM final

\begin{tabular}{|c|c|c|c|c|c|c|c|}
\hline Ítem & Pregunta & Nunca & $\begin{array}{l}\text { Pocas } \\
\text { veces }\end{array}$ & $\begin{array}{c}\text { Algunas } \\
\text { veces }\end{array}$ & $\begin{array}{l}\text { Muchas } \\
\text { veces }\end{array}$ & Siempre & No sabe \\
\hline 1 & $\begin{array}{l}\text { Estamos de acuerdo en cómo deben } \\
\text { actuar los miembros de nuestra familiar }\end{array}$ & 1 & 2 & 3 & 4 & 5 & 0 \\
\hline 2 & $\begin{array}{l}\text { Estamos de acuerdo en las cosas que } \\
\text { son importantes para nuestra familia }\end{array}$ & 1 & 2 & 3 & 4 & 5 & 0 \\
\hline 3 & $\begin{array}{l}\text { Sabemos qué queremos lograr como } \\
\text { familia en el futuro }\end{array}$ & 1 & 2 & 3 & 4 & 5 & 0 \\
\hline 4 & $\begin{array}{l}\text { Intentamos mirar el lado positivo de } \\
\text { las cosas }\end{array}$ & 1 & 2 & 3 & 4 & 5 & 0 \\
\hline 5 & $\begin{array}{l}\text { Intentamos olvidar nuestros problemas } \\
\text { por un tiempo cuando parecen que son } \\
\text { insuperables }\end{array}$ & 1 & 2 & 3 & 4 & 5 & 0 \\
\hline 6 & $\begin{array}{l}\text { Cuando hay un problema logramos ver } \\
\text { los aspectos positivos y negativos }\end{array}$ & 1 & 2 & 3 & 4 & 5 & 0 \\
\hline 7 & $\begin{array}{l}\text { Cada uno de nosotros en la familia es } \\
\text { capaz de escuchar las dos versiones de } \\
\text { una historia }\end{array}$ & 1 & 2 & 3 & 4 & 5 & 0 \\
\hline 8 & $\begin{array}{l}\text { En nuestra familia tenemos al menos un } \\
\text { día en que realizamos alguna actividad } \\
\text { todos juntos }\end{array}$ & 1 & 2 & 3 & 4 & 5 & 0 \\
\hline 9 & $\begin{array}{l}\text { Podemos pedir ayuda a alguien de afue- } \\
\text { ra de nuestra familia si lo necesitamos }\end{array}$ & 1 & 2 & 3 & 4 & 5 & 0 \\
\hline 10 & $\begin{array}{l}\text { Podemos confiar en el apoyo de los } \\
\text { demás cuando algo va mal }\end{array}$ & 1 & 2 & 3 & 4 & 5 & 0 \\
\hline 11 & $\begin{array}{l}\text { A nuestros amigos o familiares les gusta } \\
\text { visitarnos }\end{array}$ & 1 & 2 & 3 & 4 & 5 & 0 \\
\hline 12 & $\begin{array}{l}\text { Hacemos un esfuerzo por ayudar a } \\
\text { nuestros parientes cuando lo necesitan }\end{array}$ & 1 & 2 & 3 & 4 & 5 & 0 \\
\hline 13 & $\begin{array}{l}\text { Nuestros amigos y familiares nos ayuda- } \\
\text { rán si lo necesitamos }\end{array}$ & 1 & 2 & 3 & 4 & 5 & 0 \\
\hline
\end{tabular}

estimación de riesgo relativo (OR) de la cohorte de familias con mayor vulnerabilidad vs menor vulnerabilidad familiar respecto de la evolución clínica de las condiciones estudiadas. El estimador obtenido fue de 1,83 (95\% IC: 1,101-3,029). Este estimador implica que las familias que mediante las Escala SALUFAM fueron detectadas como de mayor vulnerabilidad (con un punto de corte de 3,7 ) tuvieron $83 \%$ más de riesgo de empeorar su condición a los 9 meses de aplicado el instrumento. La Tabla 3 presenta la escala SALUFAM final. La replicabilidad test-re-test obtenida mediante índice de correlación de Pearson fue de 0,84.

\section{Discusión}

La relevancia de este estudio está dada por la oportunidad de contar con un instrumento simple y de alta validez clínica concurrente y predictiva que permite valorar adecuadamente la vulnerabilidad familiar en salud. La aplicación de este instrumento puede colaborar con la aplicación clínica del enfoque familiar en la APS de nuestro país.

Los diversos instrumentos de valoración familiar existentes no han sido diseñados, en general, para ser aplicados como tamizaje en la atención primaria y carecen de validez clínica predictiva ${ }^{12,14}$ 
o, como en el caso del APGAR familiar, presentan una alta variabilidad (baja replicabilidad test- retest) en el tiempo ${ }^{15}$. Otros instrumentos como el tradicional genograma ${ }^{28}$ o Ecomapa ${ }^{29}$ tienen utilidad como complemento diagnóstico o terapéutico en intervenciones familiares pero no son de utilidad en el tamizaje de vulnerabilidad familiar. El instrumento SALUFAM destaca por su alta validez de contenido y validez de criterio concurrente reflejada en su alta sensibilidad y especificidad. Además, presenta una significativa validez de criterio predictiva, ausente en los instrumentos previamente mencionados.

El perfil clínico de la muestra refleja la situación de los pacientes adscritos a la atención primaria donde predomina un porcentaje relativamente alto de poblaciones sin un control adecuado de enfermedades tales como diabetes, asma, depresión o trastornos conductuales. De esta forma, la utilidad más directa del instrumento SALUFAM es precisamente en aquellas poblaciones de pacientes con algún grado de descompensación o dificultad en el manejo clínico de su enfermedad.

Las dimensiones de Acuerdo y Apoyo contenidas en el instrumento SALUFAM han estado consistentemente presentes en múltiples instrumentos de valoración familiar existentes ${ }^{12,14}$ y se han asociado a la evolución de enfermedades clínicas frecuentes en $\mathrm{APS}^{30-33}$. El acuerdo y el apoyo familiar son características vinculadas a la capacidad adaptativa, flexibilidad, tolerancia y negociación en los sistemas familiares. Estos son factores esenciales en la definición de familias "saludables", "fuertes" o "resilientes" y son además intervenibles ${ }^{34,35}$.

Este estudio posee limitaciones que son importantes de considerar. En primer lugar, el estudio fue realizado en una muestra de familias de bajo nivel socioeconómico y que pueden no representar apropiadamente a familias de nivel socioeconómico medio-alto o de comunidades rurales. Sin embargo, la muestra estudiada puede reflejar a una gran proporción de familias chilenas urbanas de nivel socioeconómico bajo que son las usuarias tradicionales de los centros de atención primaria del país. La comprensión del instrumento por este grupo de estudio representó un desafío mayor en la validación del instrumento. En segundo lugar, la validación del instrumento no permite sacar conclusiones para condiciones clínicas específicas. Se trata, de un instrumento genérico que fue probado en un conjunto diverso de condiciones clínicas que representan un amplio espectro de problemas de salud frecuentes de la atención primaria chilena.

En conclusión, este estudio presenta el diseño y validación del instrumento SALUFAM que permite identificar con precisión y capacidad predictiva familias de variada vulnerabilidad en salud. Este instrumento puede colaborar a mejorar la implementación del enfoque familiar y beneficiar a un conjunto de pacientes usuarios de la atención primaria en Chile.

\section{Referencias}

1. Starfield B, Shi L, Macinko J. Contribution of Primary Care to Health Systems and Health. The Milbank Quarterly 2005; 83: 457-502.

2. Pasarín MI, Berra S, Rajmil L, Solans M, Borrell C, Starfield B. A Tool to Evaluate Primary Health Care From the Population Perspective. Aten Primaria 2007; 39: 395-401.

3. Kim-Cohen J, Moffitt TE, Taylor A, Pawlby SJ, Caspi A. Maternal Depression and Children's Antisocial Behavior. Nature and Nurture Effects. Arch Gen Psychiatry 2005; 62: 173-81.

4. Shaw DS, Dishion TJ, Supplee L, Gardner F, Arnds K. Randomized Trial of a Family-Centered Approach to the Prevention of Early Conduct Problems: 2-Year Effects of the Family Check-Up in Early Childhood. J Consult Clin Psychol 2006; 74: 1-9.

5. Garber J, Clarke GN, Weersing VR, Beardslee WR, Brent DA, Gladstone TR, et al. Prevention of depression in atrisk adolescents: a randomized controlled trial. JAMA 2009; 301: 2215-24.

6. Chen E, Chim LS, Strunk RC, Miller GE. The Role of the Social Environment in Children and Adolescents with Asthma. Am J Respir Crit Care Med 2007; 176: 644-9.

7. Barth J, Schneider S, Von Känel R. Lack of Social Support in the Etiology and the Prognosis of Coronary Heart Disease: A Systematic Review and Meta-Analysis. Psychosom Med 2010; 72: 229-38.

8. García-Huidobro D, Bittner M, Brahm P, Puschel K. Family intervention to control type 2 diabetes: a controlled clinical trial. Fam Pract 2011; 28: 4-11.

9. Mc Daniel S, Campbell Th, Hepworth J, Lorenz A. Family Oriented Primary Care. $2^{\text {nd }}$ ed. New York: Springer; 2005.

10. Hidalgo CG, Carrasco E. Salud familiar: un modelo de atención integral en la atención primaria. Santiago, Chile: Ediciones Universidad Católica de Chile; 1999. 
11. Zegers B, Larraín ME, Polaino-Lorente A, Trapp A, Diez I. Validez y confiabilidad de la versión española de la escala de Cohesión y Adaptabilidad Familiar (CAF) de Olson, Russell \& Sprenkle para el diagnóstico del funcionamiento familiar en la población chilena. Rev Chil Neuro-Psiquiatr 2003; 41:39-54.

12. Hernández A. Familia y Adolescencia: Indicadores de Salud. Manual de Aplicación de Instrumentos. Organización Panamericana de la Salud, Washington D.C: 1996. Disponible en: http://www.paho.org/Spanish/HPP/HPF/ ADOL/Family.pdf [Consultado el 11 de julio de 2011].

13. Smilkestein G. The family Apgar: A proposal for a family function test and its use by physicians. J Fam Pract 1978; 6: 1231-9.

14. Larraín ME, Zegers B, Díez I, Trapp A, Polaino-Lorente A. Validez y Confiabilidad de la Versión Española de la Escala del Estilo de Funcionamiento Familiar (EFF) de Dunst, Trivette \& Deal para el Diagnóstico del Funcionamiento Familiar en la Población Chilena. Psykhe 2003; 12: 195-211.

15. Gardner W, Nutting PA, Kelleher KJ, Werner JJ, Farley T, Stewart L, et al. Does the family APGAR effectively measure family functioning?. J Fam Pract 2001; 50: 1925.

16. Rubinstein A, Terrasa S. Medicina Familiar y Práctica Ambulatoria. $2^{\mathrm{a}}$ ed. Buenos Aires: Editorial Médica Panamericana; 2006.

17. Martín Zurro A, Cano Pérez JF. Atención Primaria. Concepto, Organización y Práctica Clínica. 6ª ed. Madrid: Elsevier; 2008.

18. Taylor R. Medicina de Familia. Principios y práctica. $6^{\mathrm{a}}$ ed. Barcelona: Masson; 2006.

19. Depaux R, Campodónico L, Ringeling I, Segovia I. En el Camino a Centro de Salud Familiar. Subsecretaría de Redes Asistenciales, Ministerio de Salud, Gobierno de Chile; 2008.

20. Louro I. Matriz de salud del grupo familiar: un recurso para el diagnóstico de la situación de salud de la familia. Rev Cubana Med Gen Integr 2004; 20: 0-0. Disponible en: http://scielo.sld.cu/scielo.php?script=sci_ arttext\&pid=S0864-21252004000300006\&lng=es. [Consultado el 11 de julio 2011].

21. Ritchie J, Spencer L. Qualitative data analysis for applied policy research. In Bryman A, Burgess R, Editors. Analysing qualitative data. London: Routledge, 1993, p. 17394.

22. Patterson, J. Families experiencing stress: I. The Fa- mily Adjustment and Adaptation Response Model: II. Applying the FAAR Model to health-related issues for intervention and research. Family Systems Medicine 1998; 6: 202-37.

23. Badia X, Roset M, Montserrat S, Herdman M, Segura A. La versión española del EuroQol: descripción y aplicaciones. Med Clin 1999; 112: 79-85.

24. Beck, AT, Ward CH, Mendelson M, Mock JE, Erbaugh J. An inventory for measuring depression. Arch Gen Psychiatry 1961; 4: 561-71.

25. Mitchell H, Senturia Y, Gergen P, Baker D, Joseph C, McNiff-Mortimer K, et al. Design and methods of the National Cooperative Inner-City Asthma Study. Pediatr Pulmonol 1997; 24: 237-52.

26. Achenbach TM, Edelbrock CS. Manual for the Child Behavior Checklist and Revised Child Behavior Profile, Burlington (Vt.): University of Vermont, Department of Psychiatry, 1991.

27. Ministerio de Salud. Guía Clínica. Examen de medicina preventiva, Santiago: MINSAL 2008. Disponible en: http://www.redsalud.gov.cl/archivos/guiasges/Guia_ EMP_100108.pdf. [Consultado el 11 de julio de 2011]

28. Rogers JC. Can Physicians Use Family Genogram Information to Identify Patients at Risk of Anxiety or Depression?. Arch Fam Med 1994; 3: 1093-8.

29. Jenson K, Cornelson B. Eco-Maps: A Systems Tool for Family Physicians. Can Fam Physician 1987; 33: 172-77.

30. Marmot M, Wilkinson R. Social Determinants of Health. Marmot M Editor. Oxford: University Press, 1999.

31. Beardslee W, Gladstone T, Wright E, Cooper A. A Family-Based Approach to the Prevention of Depressive Symptoms in Children at Risk: Evidence of Parental and Child Change. Pediatrics 2003; 112: 119-31.

32. Sawyer MG, Spurrier N, Whaites L, Kennedy D, Martin AJ, Baghurst P. The Relationship between Asthma Severity, Family Functioning and the Health-Related Quality of Life of Children with Asthma. Qual Life Res 2000; 9: 1105-15.

33. Armour TA, Norris L, Jack Jr. L, Zhang X, Fisher L. The effectiveness of family interventions in people with diabetes mellitus: a systematic review. Diabet Med 2005; 22: 1295-305.

34. Walsh F. Strengthening Family Resilience. Nichols P Editor, New York: The Guilford Press; 1998.

35. Becvar D. Families that Flourish: Facilitating Resilience in Clinical Practice. New York: Norton Press; 2006. 